Postgrad. med. J. (July 1968) 44, 497-500.

$$
\begin{aligned}
& \text { K } 31 \\
& \text { P65 } \\
& 44 \\
& \text { July-Doc. }
\end{aligned}
$$

Biological

Sciences

\title{
The pathogenesis of gallstone pancreatitis
}

\author{
J. E. TRAPNELL \\ M.D., F.R.C.S. \\ Southmead Hospital, Bristol
}

\section{Summary}

The pathogenesis of gallstone pancreatitis has been re-examined. It is concluded that there is no evidence that reflux of bile occurs: even if it does, it is not, of itself; harmful in any way. Opie's 'common channel' theory is of historical interest only and should now be firmly discarded.

Clinical studies have shown the efficacy of comprehensive biliary tract surgery in terminating the recurring pattern of attacks of pancreatitis in these patients. It is suggested that this surgery eradicates a septic focus as well as a mechanical factor and that in the majority of cases it is this infective element which is important in the pathogenesis. This working hypothesis receives strong support from established clinical facts and also from experimental studies.

IT IS Now accepted that acute pancreatitis is basically an autodigestive process but the exact mechanism of pathogenesis has been a matter of speculation over many years and various hypotheses have been suggested. A great deal of time and energy has been spent in the experimental evaluation of these various hypothetical mechanisms but, in contrast to clinical studies, very few unequivocal findings have emerged from the laboratory. Although literally thousands of dogs have lost their lives, no true model of the common forms of human pancreatitis has yet been produced and much of the work has been grossly unphysiological. Our present understanding of the whole subject has been reviewed elsewhere (Anderson \& Trapnell, 1968).

In Great Britain at the present time acute pancreatitis is not a common condition. It is, however, of considerable importance because it still carries a mortality in the region of $20 \%$. Subsequent investigation of these patients shows that approximately $60 \%$ of them have an associated biliary tract disease and it is now accepted that this represents a firm aetiological relationship. These patients, therefore, form a distinguishable subgroup of the overall disease.
Clinical studies have established the natural history of the illness and have demonstrated that it is quite distinct from the pancreatitis associated with chronic alcoholism (Howard \& Jordan, 1960; Trapnell, 1966). The aim of this communication is to re-examine the pathogenesis of this particular type of acute pancreatitis.

For 60 years it has been held that reflux of bile via a 'common channel' is the underlying mechanism (Opie, 1901) and surgical thinking has been dominated by Opie's hypothesis. This must, therefore, be re-assessed carefully and critically. Much of the evidence which has accumulated in the literature is confusing and conflicting but the following salient features have emerged.

(1) One of the first aspects of this theory to be investigated was the anatomical arrangement at the junction of the common bile duct and the duct of Wirsung and much time and energy was expended in assessing the frequency with which reflux could occur. These studies merit the following conclusions:

Firstly, all patients with pancreatitis do not have a common channel. In fact in a proportion-perhaps $50 \%$-direct biliary reflux is probably impossible.

Secondly, it is apparent that $50 \%$ of the population at large carry an anatomical arrangement whereby biliary reflux could occur-and yet acute pancreatitis remains an uncommon condition.

Finally, this anatomy is present throughout life, yet pancreatitis has a limited age incidence.

(2) The next question to be considered concerned the conversion of the anatomical possibility into an actuality. From clinical studies it was soon realized that while the association of gallstones with pancreatitis was frequent the creation of a diversion by impaction of a small stone at the sphincter was rare. Indeed, the size of the stone must be within narrow limits for it to occur at all, for if it is too large it will occlude the common bile duct and obstructive jaundice rather than acute pancreatitis will result. Hence to explain reflux in the absence of 
a stone the concept of spasm of the sphincter of Oddi was introduced (Archibald, 1919).

Experimental confirmation of this hypothesis was only obtained when most unphysiological pressures (of the order of $600 \mathrm{~mm}$ of water) were used whereas with low pressures the results were variable. Spasm of the sphincter of Oddi undoubtedly does occur in response to various stimuli-for example alcohol and opiates-and a rise in pressure in the biliary and pancreatic systems results (Parry, Hallenbeck \& Grindlay, 1955). Furthermore, enzyme back-diffusion is increased under these conditions for this is the basis of the various 'provocation' tests. It does not, however, follow that these changes occur as the result of biliary reflux. Indeed anatomical studies have revealed that spasm of the sphincter may close off both the common bile duct and the duct of Wirsung in the majority of individuals (Caroli et al., 1960). Hence it would seem that while spasm can and does occur it will result in obstruction of the pancreatic and common bile ducts whether or not a 'common channel' is formed.

(3) A further aspect of this problem which has been much studied concerns the pressure differences in the two duct systems, for the hypothesis requires that the pressure in the common bile duct must overcome that in the pancreatic duct. Once again, however, many of the experimental reports cannot be accepted as reflecting the physiological state, recordings having usually been made after the establishment of a fistula or in the face of obstruction (Mann \& Giordano, 1933 ; Elliott, Williams \& Zollinger, 1957). The most reliable studies have come from Parry's technique of retrograde catheterization of the bile and pancreatic ducts through the substance of the liver and the pancreas (Parry et al., 1955 ; Menguy et al., 1955). These workers have demonstrated that bile is most unlikely to flow into the duct of Wirsung except after cholecystectomy.

In addition to these experimental findings there is iittle clinical evidence that biliary reflux occurs. Direct recordings from patients are rarely possible but such reports as there are, confirm that pressures are higher in the duct of Wirsung (Anderson \& Hagstrom, 1962). In acute pancreatitis pancreatic enzymes have been found consistently in bile aspirated from the gall bladder (Popper, Necheles \& Russell, 1948), but on the other hand, biliary staining of the pancreas is rarely seen when cases of pancreatitis have been explored in the acute stages (Priestly, 1955 ; Trapnell, 1966). Staining of the pancreatic duct is also an uncommon finding at necropsy in patients dying of acute pancreatitis and in any event it may be argued that this type of reflux could result from a passive backflow facilitated by the 'shut-down' of pancreatic secretion which has been shown to occur at the onset of the disease (Grossman, 1955 ; Dreiling \& Janowitz 1962). Indeed in some patients it could even be produced during the autopsy examination itself for this appearance is observed from time to time in cases with no evidence of either pancreatic or biliary pathology. Finally, it is well recognized that the pancreatic duct is only very rarely opacified on intravenous cholangiography - that is under physiological conditions. From this evidence it would appear that the relative intra-duct pressures do not normally allow bile to enter the pancreas. This situation could theoretically be reversed after cholecystectomy but there is no clinical evidence that this does, in fact, occur.

(4) Finally, it has been shown conclusively that bile may enter the pancreatic ducts without any harmful effects ensuing (Elmslie, White \& Magee, 1966). The original hypothesis required that bile was necessary to activate trypsinogen, but the apparent proof of this view was based once again on unphysiological experimental work involving forceful injection into the pancreatic system, thus introducing the additionalo factor of acinar rupture. Recent careful studies have failed to demonstrate any tryptic activity? in the pancreatic juice after incubation with bile in vitro (Haverback et al., 1960). Furthermore, diversion of bile through the pancreatic duct, carried out experimentally in various animals, has failed to produce any evidence of acute pancreatitis (Elmslie et al., 1966) even though proteolytic activity in the main duct may be high (Robinson \& Dunphy, 1963).

It must be concluded, therefore, that there is no evidence that biliary reflux occurs: even if it does, it is not of itself harmful in any way. Current thinking is still too much bemused by Opie's original hypothesis and it is high time that it was discarded. It would however, be very unsatisfactory if the matter were to be left in this negative vacuum and a constructive alternative must be sought.

It has already been stated that the majority of pertinent facts about the aetiology of acute pancreatitis have emerged from simple clinical studies. Characteristically these patients with gallstone pancreatitis will suffer recurrent episodes so long as they retain their biliary tract disease but, in spite of this, they do not progress to chronic pancreatic insufficiency with calcification, fibrosis and duct stricture. There is thus a clear distinction from the group with an alcoholic 
aetiological basis. These recurrent episodes can be interrupted by biliary tract surgery and provided that all abnormalities are comprehensively eradicated the disease process is terminated. It is important to note, however, especially in the present context, that the majority of these patients only have stones in the gall bladder and cholecystectomy is all that is required for cure. In particular surgery in the 'common channel' region (e.g. sphincterotomy) is rarely necessary, and when it is undertaken, not only is it hazardous, but personal experience has shown that the results of this procedure are probably inferior to external choledochoduodenostomy. In the vast majority of cases, however, the anatomical arrangement is not altered and this is especially significant in view of the rise in bileduct pressure, already mentioned, which has been shown to occur experimentally after removal of the gall bladder (Menguy et al., 1955). This clinical evidence taken in conjunction with the experimental work should lead to the final rejection of the 'common channel' theory and biliary reflux as the simple cause of acute pancreatitis in these patients.

But what then does surgery actually achieve for it is clearly beneficial? In a minority of these cases a mechanical factor is present and is responsible for the development of the pancreatitis. In general terms obstruction of the duct of Wirsung may result from a variety of causes: carcinoma, oedema following sphincterotomy, the presence of a long-armed T-tube, rare conditions such as parasitic infestation or-of special concern in the present context-calculus impaction; and all of these may be associated with the development of pancreatitis. Whether or not the duct of Wirsung is blocked in continuity with the common bile duct or separately by a larger stone occluding both channels, a rise in intra-duct pressure with increased back diffusion will result and this is considered to play a major part in the pathogenesis of the pancreatitis which ensues (Anderson \& Trapnell, 1968). Anatomical features will, therefore, dictate the clinical outcome of calculus impaction for either obstructive jaundice or acute pancreatitis or both may occur, the final result depending both on the local anatomy and on the size of the calculus itself. Operation removes the obstruction but it should be stressed that such cases form only a small percentage in the overall group under consideration.

The majority of patients do not have stones in the common bile duct, yet elimination of biliary tract disease still alters the natural history of the pancreatitis. Indeed in four personal cases removal of a stone-bearing gall bladder has interrupted recurrences in patients shown to have a duct of Wirsung opening into the duodenum separately from the common bile duct. A remission has also been achieved on a number of occasions following the removal of a shrunken stone-free gall bladder remnant which had previously been decompressed by emergency cholecystostomy. Mechanical factors cannot have played any part in either of these situations and some other explanation must therefore be sought. Although the formation of gallstones is probably not primarily an infective process, secondary infection does occur, and the possibility must now be considered that this could be a factor in the pathogenesis.

The first details of acute pancreatitis were nearly all described from necropsy findings, and infection in and around the necrotic gland was commonly noted. This coupled with the presence of gallstones soon led to the suggestion that the pancreatitis might be infective in origin in a proportion of cases. Various routes of bacterial spread to the gland were considered but none of these received support for long and in 1927 Kaufmann concluded that this form of direct infection was "inadmissable as an aetiological basis for acute pancreatitis'. There are, however, some fragments of experimental work which suggest that infection, especially in the biliary tree, could damage the gland in a more indirect way.

In 1954 Thal \& Brackney reported a piece of experimental work which has received little further attention. These workers were able to produce pancreatitis by a form of Schwartzmann reaction. In a well designed experiment they perfused the pancreatic ducts of rabbits with $E$. coli endotoxin at low pressure, and then $24 \mathrm{hr}$ later were able to induce acute pancreatitis by intravenous injection of the same toxin. All the rabbits died with haemorrhagic necrosis of the gland. In control experiments if either the sensitizing or provocative dose were omitted the pancreatitis did not develop. The suggestion is now put forward that this may be the mechanism in some cases of acute pancreatitis associated with infection in the biliary tract. The route of sensitization must remain hypothetical, but may perhaps be by biliary reflux. This would certainly be supported by the common clinical finding of sparing of the tail gland-the proximal part being more accessible to perfusion. Bergkvist's (Bergkvist \& Seldinger, 1958) radiological findings that reflux was rarely 'total' adds further weight on this point. Provocation could occur during the toxaemia of acute cholecystitis-associated with 
pancreatitis in $15 \%$ of cases (Ivy \& Gibbs, 1952) and in the remainder perhaps as the result of an otherwise sub-clinical exacerbation or transient cystic duct obstruction producing a bacteraemia. The hypothesis receives further support from the work of Anderson, Mehn \& Method (1959) who were studying the early histological changes in experimentally induced pancreatitis. They observed that the first structures to be affected were the arterioles and that the changes were similar to those described in the hypersensitivity reaction. Finally, Cotlar and his co-workers (1960), using as an experimental model perfusion of the pancreatic duct with staphylococcal toxin, demonstrated that the pancreatitis so produced was not modified by penicillin. However, when steroids were given the changes were much less severe. These workers concluded that they were inhibiting a sensitivity phenomenon.

This hypothesis, though purely tentative, is strongly supported by clinical observations and in particular by the results of surgery. Mention has already been made of the benefit derived from the removal of a shrunken remnant of gall bladder, previously decompressed by cholecystostomy. This is usually stone-free but may still be a septic focus. Then again the theory affords an explanation for the small but interesting group of cases with recurrent attacks of pancreatitis after cholecystectomy, who were found to have a dilated common duct but no residual stones and who were cured by choledochoduodenostomy (Trapnell, 1966). Presumably the dilatation of the duct resulted from an incomplete obstruction-not sufficient to produce jaundice but enough to allow stasis with secondary infection. That infection was present in the common bile duct was further borne out by its white and thickened appearance: frank inflammation of the mucosa being evident on three occasions. Choledochoduodenostomy by decompressing the biliary tree promoted free drainage in these cases and the attacks of pancreatitis ceased, not because of diversion of bile, but because the infection was removed. Finally, this mechanism would account too, for the beneficial effect of removal of residual common duct stones. These may present with recurrent pancreatitis rather than with jaundice - the result of infection rather than obstruction.

\section{References}

Anderson, M.C., Mehn, W.H. \& Method, H.L. (1959) Treatment of acute haemorrhagic pancreatitis with adrenocorticosteroids. Arch. Surg. 78, 802.
Anderson, M.C. \& Hagstrom, W.J. (1962) A comparison of pancreatic and biliary pressures recorded simultaneously in man. Can. J. Surg. 5, 461.

Anderson, M.C. \& Trapnell, J.E. (1968) Mechanisms responsible for the initiation and progression of acute pancreatitis. (In press).

Archibald, E. (1919) The experimental production of pancreatitis in animals as a result of the resistance of the common duct sphincter. Surg. Gynec. Obstet. 28, 529.

Bergkvist, A. \& Seldinger, S.I. (1958) Pancreatic reflux in operative cholangiography in relation to pre- and postoperative pancreatic affection. Acta chir. scand. 114, 191.

Caroli, J., Porcher, P., Pequignot, G. \& Delattre, M. (1960) Contribution of cineradiology to the study of the function of the human biliary tract. Amer. J. dig. Dis. $5,677$.

Cotlar, A.M., Hudson, T.L., Kaplan, M.H. \& Cohn, I. (1960) Experimental haemorrhagic pancreatitis produced by staphylococcal toxin. Surgery, 47, 587.

Dreiling, D.A. \& JANOwitz, H.D. (1962) The measurement of pancreatic secretory function. The Exocrine Pancreas, Ciba Foundation Symposium, p. 225. Churchill, London.

ElliotT, D.W., Williams, R.D. \& ZollingeR, R.M. (1957) Alterations in the pancreatic resistance to bile in the pathogenesis of acute pancreatitis. Ann. Surg. 146, 669.

Elmslie, R., White, T.T. \& Magee, D.F. (1966) The significance of reflux of trypsin and bile in the pathogenesis of human pancreatitis. Brit. J. Surg. 53, 809.

Grossman, M.I. (1955) Experimental pancreatitis. Arch. intern. Med. 96, 298.

Haverback, B.J., Dyce, B., Bundy, H. \& Edmondson, H.A. (1960) Trypsin, trypsinogen and trypsin inhibitor in human pancreatic juice. Amer. J. Med. 29, 424.

HowARD, J.M. \& JoRdAN, G.L. (1960) Surgical Diseases of the Pancreas. Lippincott, Philadelphia.

Ivy, A.C. \& GibBs, G.E. (1952) Pancreatitis-a review. Surgery, 31, 614.

Kaufmann, M. (1927) An experimental study of the lymphatic theory of pancreatitis. Surg. Gynec. Obstet. 44, 15.

ManN, F.C. \& Giordano, A.S. (1923) The bile factor in pancreatitis. Arch. Surg. 6, 1.

Menguy, R.B., Hallenbeck, G.A., Bollman, J.L. \& GrINDLAY, J.H. (1955) Pancreatic and biliary intraductal pressures. Surg. Forum, 6, 539.

OpIE, E.L. (1901) The etiology of acute haemorrhagic pancreatitis. Bull. Johns Hopk. Hosp. 12, 182.

Parry, E.W., Hallenbeck, G.A. \& Grindlay, J.H. (1955) Pressures in the pancreatic and common ducts. Arch. Surg. 70, 757.

Popper, H.L., Necheles, H. \& Russell, K.C. (1948) Transition of pancreatic oedema into pancreatic necrosis. Surg. Gynaec. Obstet. 87, 79.

Priestly, J.T. (1955) Discussion on 'acute inflammation of the pancrease'. Arch. intern. Med. 96, 321.

Robinson, T.M. \& DunPhy, J.E. (1963) Continuous perfusion of bile and protease activators through the pancreas. J. Amer. med. Ass. 183, 530.

Thal, A.P. \& Brackney, E. (1954) Acute haemorrhagic pancreatic necrosis produced by a local Schwartzmann reaction. J. Amer. med. Ass. 155, 569.

Trapnell, J.E. (1966) The natural history and prognosis of acute pancreatitis, M.D. Thesis. University of Cambridge. 